\title{
Mechanical and mathematical model for estimating the wear rate of the side face of rails
}

\author{
Nikolay Karpushchenko ${ }^{1, *}$, and Pavel Trukhanov \\ ${ }^{1}$ Siberian Transport University, Dusi Kovalchuk st., 191, 630049, Novosibirsk, Russia
}

\begin{abstract}
The paper presents a mechanical and mathematical model for estimating the wear of rails in the form of a generalized indicator called the wear factor, which includes the total relative sliding caused by turning the truck when entering the curve and rotating the wheel. The wear factor also includes the normal pressure at the point of contact between the wheel flange and rail, the contact area, the friction coefficient, and the coefficient that takes into account the wobble of wheel sets.
\end{abstract}

\section{Introduction}

The wear of the side surface of the rail head and wheel flange is an important problem associated with a reduction in the operating costs of railways. The problem arose due to the increase in axial loads, the exploitation of new types of rolling stock. Study of this problem has shown the need for systematic monitoring of the state of the track, rolling stock and refinement of the model of interaction in the wheel-rail system when moving along curvilinear sections of the track. The improved interaction technique allows analyzing the impact of ongoing measures to reduce the intensity of wear and to predict their effectiveness. Numerous studies on the wear of the side surface of the rail propose different expressions containing the angle of attack of wheel on the rail, the magnitude of the guiding force, and the coefficient of friction as the wear factor [1-2]. However, these studies did not take into account the sliding of the flange along the side face of the rail caused by the rotation of the wheel. This was first noticed by A.Ya. Kogan in the study [3].

In this paper, the properties of the mathematical curve - cycloids - are used to study the sliding process of the wheel flange along the side face of the rail [4].

\section{Formulation of the problem}

To estimate the influence of the parameters of the rail track and the trucks of the rolling stock on the wear of the rails, we use an indicator called the wear factor in the following interpretation:

$$
F_{\text {wear }}=\frac{N u_{\mathrm{M}}^{0} k_{\mathrm{w}} \mu}{A_{0}},
$$

\footnotetext{
* Corresponding author: kni@stu.ru
} 
where $N$ - normal pressure at the point of contact between the wheel flange and rail;

$u_{\mathrm{M}}^{0}$ - relative sliding of the wheel flange on the rail;

$k_{\mathrm{w}}$ - coefficient that takes into account the wobble of wheel sets;

$\mu$ - coefficient of friction;

$A_{0}$ - reduced contact area of wheel and rail.

\section{Passing of the train along the curve}

When the rolling stock passes along the curve, a constantly acting transverse force appears, turning the train to the required angle, so it is in a complex reciprocating motion.

In this regard, the movement of the truck in the plane of the track can be represented as its rotation relative to the center of the curve (point A in Fig. 1) with the angular velocity $\Omega=\frac{V}{R}$, and also the translational motion with the velocity $V$ of its some point $O$, which is the turning pole lying at the base of the perpendicular dropped from the center of the curve $A$ to the longitudinal axle of the truck, and the simultaneous rotation of the truck around this pole with the same angular velocity $\Omega$.

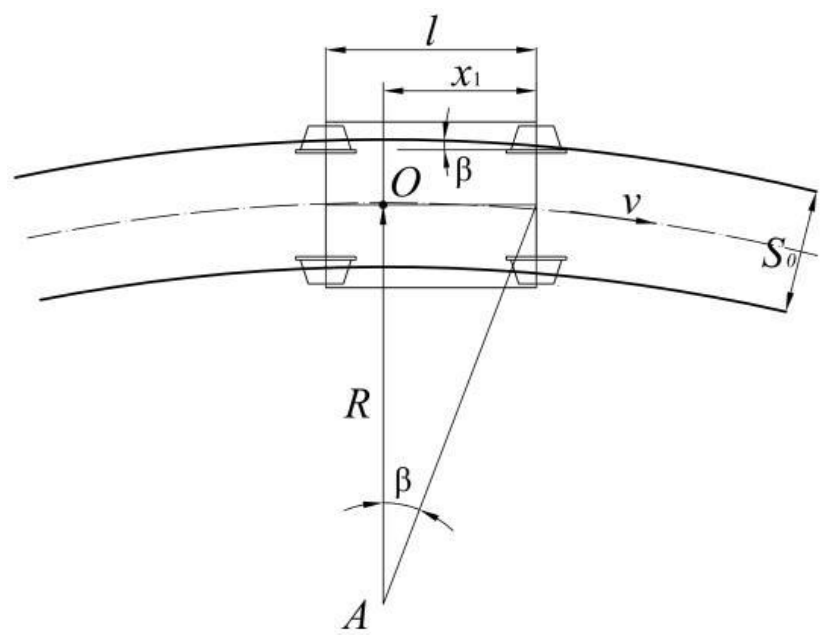

Fig. 1. The kinematic scheme of rotation of the train around the center of the curve and around the pole of rotation $O$ when it passes around a circular curve.

Due to the fact that the railway train has more than one axle, and within the rigid base they are all parallel to each other, the movement associated with the turn of a truck is possible only when the wheels slide on the rails. The process of passing of the train along a curved section is called "circumscribing". The main parameters of the rail track, on which the character of the passing depends, are the radius of the curve $R$, the superelevation of the outer rail, and the rigid base of the train $l$.

The absolute velocity of movement of the points of contact between the wheels and the rails is determined as the sum of their translational velocity in the rotational motion of the train around the vertical axis $A$ that passes through the center of the curve (Figure 1) and relative velocity in the rotational motion of the wheel in the frame of the truck around its horizontal axis $O_{1}$ (Figure 2). 


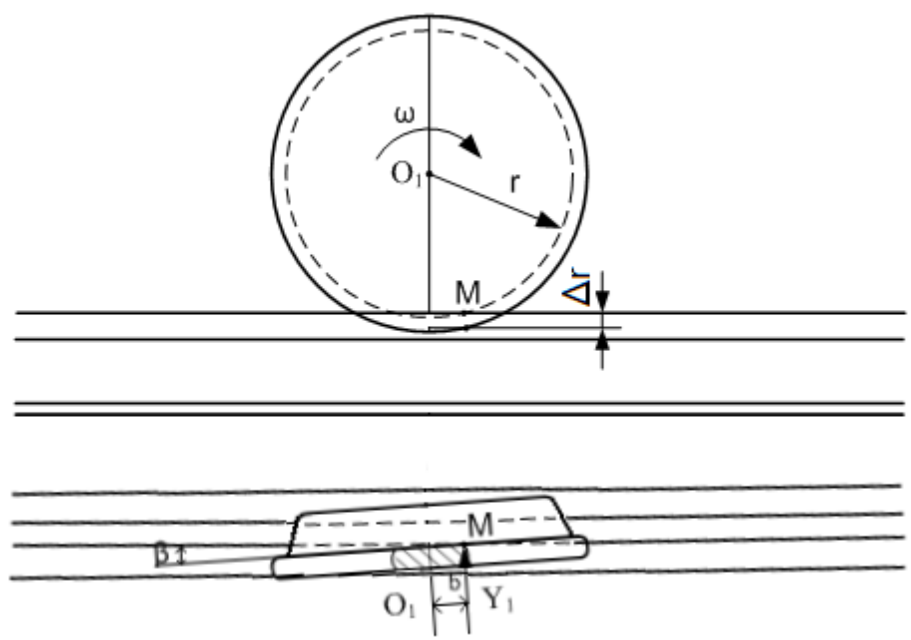

Fig. 2. The scheme of attack of the wheel flange on the rail.

Let us denote the angular velocity of the first rotational motion of the truck $\Omega$ and the wheels $\omega$. The translational velocity of the train $V$ along the curve of radius $R$ will be:

$$
V=\Omega R=\omega r .
$$

The problem of the value and direction of wheel sliding is solved if the following indicators are known: the position of the rotation pole of the train $O$ (figure 1), its speed $V$, the angular velocity of the wheel set rotation around the axle $\omega$, and $r_{\mathrm{o}}$ and $r_{\mathrm{i}}$ are the radii of the outer and inner wheels in their contacts with rails. In this case, the value of the transverse sliding on both wheels of the wheel set is:

$$
u_{\mathrm{y}}=\frac{\Omega x_{1}}{\cos \gamma}
$$

where $\Omega$ - angular velocity of rotation of the train around the center of the curve;

$x_{1}$ - abscissa of the train axis in relation to the turning pole;

$\gamma$-slope of the working surface of the wheel flange to the plane of the track.

If the velocity of the train along the center line of the curved track section is equal to $V=\Omega R$ and it is assumed that the wheel passing along the inner rail of the curve (radius $r_{\mathrm{i}}$ ) rolls without sliding, then the angular velocity of rotation of the wheel set is equal to $\frac{\Omega R}{r_{i}}$.

The surface velocity of the wheel of radius $r_{\mathrm{o}}$ passing along the outer rail (with sliding) will be equal to $\frac{\Omega R r_{o}}{r_{i}}$.

The value of the relative transverse sliding of the wheel along the outer rail will be:

$$
u_{\mathrm{y}}^{0}=\frac{x r_{i}}{R r_{0} \cos \gamma} \text {. }
$$

\section{Sliding of a rotating wheel flange along the side face of the rail head}


To study the sliding processes of the wheel flange along the side face of the rail, the properties of the mathematical curve - cycloids - are used. Cycloids are curves circumscribed by a point of a circle rolling without sliding along the guiding curve $L$ (the abscissa axis). If $C$ is the center of a circle and $M$ is a point on the connecting segment $C M$ ( $M$ is a point of the circle), then $M$ will circumscribe a curtate cycloid. If $M$ lies outside the circle on the extension of $C M$, then $M$ describes a prolate cycloid. The prolate and curtate cycloids are also called trochoids [4].

Prolate cycloid (Figure 3). Equations in parametric form

$$
\left.\begin{array}{l}
x=r(\alpha-\lambda \sin \alpha), \\
y=r(1-\lambda \cos \alpha) .
\end{array}\right\},
$$

where $r$ - radius of a circle, $\lambda=\frac{r+\Delta r}{r}$.

$\alpha=\angle M C_{1} P$ (oscillation angle), $\lambda r=C_{1} M$ (when $\lambda>1-$ prolate cycloid, when $\lambda<1-$ curtate cycloid). Peaks: $A_{k}((2 k-1) \pi r,(1+\lambda) r)$. Dips: $\left.B_{\kappa}(2 \kappa \pi r,(1-\lambda) r)\right)$.

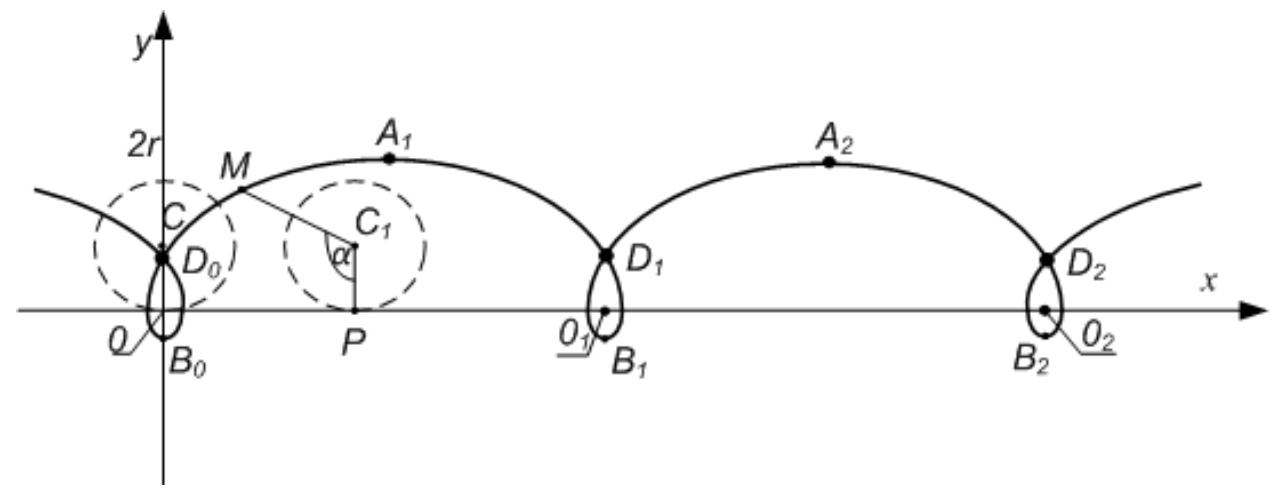

Fig. 3. Prolate cycloid.

When $\alpha$ varies from zero to $2 \pi$, the point will circumscribe the arc depicted in Figure 3 . The entire curve corresponding to a change of $\alpha$ from $-\infty$ to $+\infty$ consists of an infinite set of such arcs.

Tangent and normal to the cycloid. The sliding velocity modulus of the point $M$ located on the wheel flange will be equal to the product of the normal $n_{M}$ to this point and the angular velocity of the wheel rotation $\omega$ :

$$
u_{\mathrm{M}}=n_{\mathrm{M}} \omega \text {. }
$$

In this regard, it is necessary to determine the equations of the tangent and the normal to the point $M$. The curve given by the explicit equation $y=\mathrm{f}(x)$ has a tangent at each of its points $(\mathrm{x}, \mathrm{y})$ (Figure 4), the slope ratio tge of which is expressed by the formula:

$$
\operatorname{tg} \varepsilon=y_{\mathrm{x}}^{\prime}=f^{\prime}(x)
$$

The slope ratio of the tangent to the curve given in the parametric form can be expressed as follows:

$$
\operatorname{tg} \varepsilon=y_{x}^{\prime}=\frac{d y}{d x}=\frac{\frac{d y}{d t}}{\frac{d x}{d t}}=\frac{y^{\prime} t}{x^{\prime} t}
$$


Let us consider the problem of drawing a tangent to the cycloid given by formulas (5) (Fig. 4).

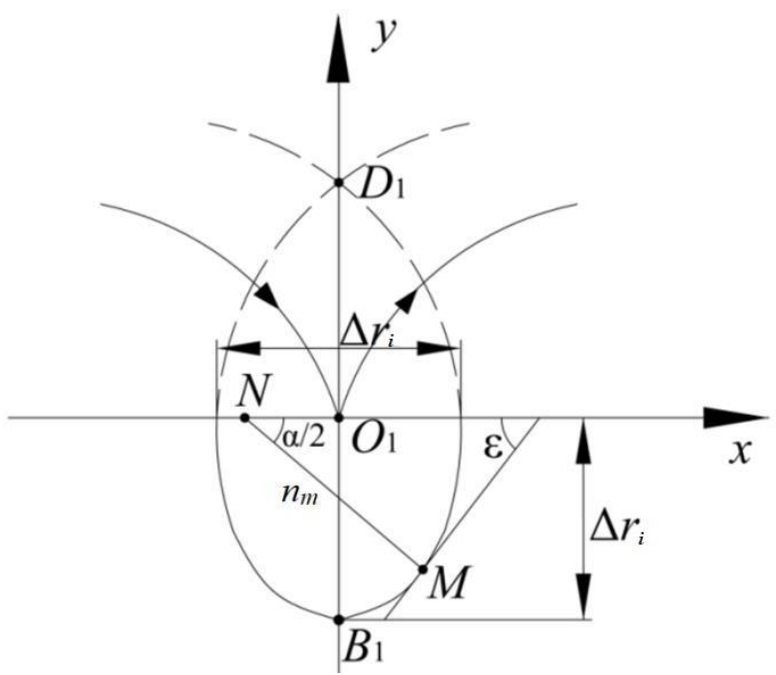

Fig. 4. A motion trajectory of the point $M$ relative to the side face of the rail head.

In this case:

$$
\left.\begin{array}{l}
x^{\prime}(\alpha)=r(1-\lambda \cos \alpha) \\
y^{\prime}(\alpha)=r \lambda \sin \alpha .
\end{array}\right\}
$$

Whence in accordance with formula (8) we obtain:

$$
\operatorname{tg} \varepsilon=\frac{\lambda \sin \alpha}{1-\lambda \cos \alpha} .
$$

For ordinary cycloid

$$
\text { and } \varepsilon=\frac{\pi-\alpha}{2} \text {. }
$$

The normal is a straight line perpendicular to the tangent at the point of tangency $M$. The positive direction of the tangent points toward the positive direction of the curve, and the positive direction of the normal is obtained by rotating the positive tangent direction by $90^{\circ}$ counterclockwise.

The segment of the normal $M N$ (Figure 4) before crossing with the $x$-axis in Cartesian coordinates $M N=\left|y \sqrt{1+y_{x}^{\prime 2}}\right|$.

In the parametric form, the segment of normal $M N$ is equal to:

$$
n_{M}=r(1-\lambda \cos \alpha) \sqrt{1+\left(\frac{\lambda \sin \alpha}{1-\lambda \cos \alpha}\right)^{2}}=r \sqrt{1+\lambda^{2}-2 \lambda \cos \alpha} .
$$

After the transformation of expression (12), taking $2 \lambda \approx 1+\lambda^{2}$ we obtain: 


$$
n_{M}=2 r \sqrt{\lambda} \sin \frac{\alpha}{2}
$$

\section{Relative sliding of the wheel flange over the rail head}

The velocity vector of the point $M$ located on the wheel flange (Figure 4 ) is tangential to the trajectory of the prolate cycloid, and the sliding velocity modulus of the point $M$ is equal to the product of the normal to this point and the angular velocity of the wheel rotation $\omega$.

$$
u_{\mathrm{M}}=n_{\mathrm{M}} \omega=2 r \sqrt{\lambda} \sin \frac{\alpha}{2} \omega .
$$

The relative velocity of the point $M$ along the cycloid trajectory is:

$$
u_{\mathrm{M}}^{0}=\frac{u_{\mathrm{M}}}{\omega r}=2 \sqrt{\lambda} \sin \frac{\alpha}{2} .
$$

Projections of the relative sliding of the point $M$ on the longitudinal and vertical axes $x$ and $z$ in the plane of the wheel flange will be:

$$
\left.\begin{array}{l}
u_{M x}^{0}=2 \sqrt{\lambda} \sin \frac{\alpha}{2} \cos \frac{\alpha}{2} \\
u_{M z}^{0}=\frac{2 \sqrt{\lambda} \sin ^{2} \frac{\alpha}{2}}{\sin \gamma} .
\end{array}\right\}
$$

The slope in accordance with formula (10):

$$
\varepsilon=\operatorname{arctg}\left(\frac{\lambda \sin \alpha}{1-\lambda \cos \alpha}\right)
$$

Projections of the total relative sliding of the point $M$ located on the flange of the wheel that attacks the outer rail on the $x, y, z$ axis are:

$$
\left.\begin{array}{l}
u_{x}^{0}=-2 \sqrt{\lambda} \sin \frac{\alpha}{2} \cos \frac{\alpha}{2} \\
u_{y}^{0}=\frac{x r_{i}}{R r_{0} \cos \gamma} ; \\
u_{z}^{0}=\frac{2 \sqrt{\lambda} \sin ^{2} \frac{\alpha}{2}}{\sin \gamma} .
\end{array}\right\}
$$

The modulus of the total relative sliding of the point $M$ on the wheel flange will be:

$$
u_{M}^{0}=\sqrt{\left(-2 \sqrt{\lambda} \sin \frac{\alpha}{2} \cos \frac{\alpha}{2}\right)^{2}+\left(\frac{x r_{i}}{R r_{0} \cos \gamma}\right)^{2}+\left(\frac{2 \sqrt{\lambda} \sin ^{2} \frac{\alpha}{2}}{\sin \gamma}\right)^{2}} .
$$


According to the obtained dependence, the relative sliding of the point $M$ located on the wheel flange depends on the angle $\alpha$, which characterizes the position of the normal to this point.

The angle $\alpha$ can be determined from the equation of abscissa of the point $M$ (5), equating $x=b$ (contact prediction), we obtain:

$$
b=r(\alpha-\lambda \sin \alpha) .
$$

\section{Transverse forces acting on the outer rail of the curve when circumscribing a truck of model 18-100}

The normal pressure $N$ at the point of contact between the wheel flange and the rail, taking into account the frictional force $\mu N$ occurring in the contact, will be:

$$
N=\frac{Y_{1}}{(\sin \gamma-\mu \cos \gamma)},
$$

where $Y_{1}$ - guiding force acting on the first axle of the truck;

$\gamma$-slope of the working surface of the flange to the plane passing through the top of the rail heads.

In determining the transverse forces, the calculation scheme is adopted, when both trucks of the car are guided by the first axes along the outer rail.

The balance equations of the truck in the plane of the track include: the guiding force $Y_{1}$ from the action of the rail to the flange of the attacking wheel, the frictional force, and the centrifugal force, the transverse component of the longitudinal force $\Delta F$ occurring in the passage of car along the curve and applied to the center plate $[5,6]$.

In this scheme, the value of the guiding force $Y_{1}$ for the truck of a freight car of model $18-100$ is determined from equation:

$$
Y_{1}=3 P_{k} \mu k_{w} k_{R}+\Delta F+8,1+0,3 P_{k} \alpha_{v} .
$$

where $P_{k}$ - the wheel load on the rail.

Formula (1) is valid only when the flanges touch the side face of the rail, which occurs when circumscribing of trucks of model 18-100 into curves with a radius of $600 \mathrm{~m}$ or less. At large radii, due to the wobble of wheel sets, this contact is disrupted the more often, the larger the radius of the curve. We can assume that there is practically no such contact in the curves with $\mathrm{R}>1000 \mathrm{~m}$.

This can be taken into account by introducing into the formula (22) a coefficient that takes into account the contacting probability at the considered point $M$ :

$$
k_{\mathrm{w}}=2 \cdot 10^{-3}(1000-R) \text {. }
$$

Theoretical and experimental studies have established the influence of the curvature of the track on the transverse forces of interaction between wheels and rails in the curves [7, 8]. In this regard, it is necessary to introduce into formula (22) a coefficient that takes into account the increase in the guiding forces with decreasing radius of the curves:

$$
k_{R}=\left(\frac{400}{R}\right)^{0,175} \text {. }
$$


The value of the transverse unbalanced acceleration $\alpha_{\mathrm{u}}$ in formula (22) depends on the velocity $v$, the radius of the curve $R$, the superelevation of the outer rail $h$, and is determined by the formula:

$$
\alpha_{v}=\frac{v^{2}}{13 R}-0,0061 h
$$

Calculations on the circumscribing of railway trains into curves on the basis of the generalized method [5] with allowance for the arising longitudinal compressive forces were performed in $[7,9]$. The values of the transverse components of the longitudinal forces $F$ applied to the truck center plates in the curve are determined by the formula:

$$
\Delta F=\frac{3600 F}{3600-F}\left(0,028 \pm \frac{6,895}{R}\right)
$$

where $R$ - radius of a circular curve.

The contacting of the wheels flanges with rails was studied by experimental methods in curves under the passing rolling stock, and also in laboratory conditions with full-scale samples of wheels and rails.

The contact area of the wheel flange and the side face of the rail is determined depending on the angle of attack $\beta$ [9]:

$$
A_{0}=68+2035 \operatorname{tg} \beta\left(\mathrm{mm}^{2}\right)
$$

When calculating using the wear factor (1), the influence of the contact area on the wear rate is taken into account in relative units:

$$
A_{0}=1+30 \operatorname{tg} \beta=1+\frac{30 x_{1}}{R} .
$$

According to the obtained dependence, the relative sliding of the point $M$ located on the wheel flange depends on the angle $\alpha$, which characterizes the position of the normal to this point.

The angle $\alpha$ can be determined from equation (20) of the abscissa of the point $M$ on the wheel flange, taking $x=b$, we obtain:

$$
x=b=r(\alpha-\lambda \sin \alpha) .
$$

The distance $b$ from the geometric axis of the first wheel set to the point of contact of the wheel flange with the rail (contact prediction) is determined by the formula:

$$
b=\frac{x_{1} r \operatorname{tg} \gamma}{R},
$$

where $r$-radius of a car wheel, $r=0,475 \mathrm{~m}$;

$\gamma$-slope of the working surface of the flange to the plane of the track. For the new car wheel $\gamma=60^{\circ}$, for worn one $\gamma=65^{\circ} \ldots 70^{\circ}$.

After calculating the value of $b$, we proceed to determine the angle $\alpha$ from the dependence by the method of successive approximation:

$$
\frac{b}{0,475}=\alpha-\lambda \sin \alpha \text {. }
$$

To do this, take as initial value $\alpha=0.5$ radian and then change its value up and down in steps of 0.001 radian, until the right side of dependence (30) is equal to the left. 


\section{Calculation of the wear rate}

According to the algorithm presented in the paper, a computer program was developed and a wear factor calculation was made (table). Variable parameters in the calculation were: $R$ radius of the curve; $P_{\mathrm{w}}$ - wheel load to rail; $F$ - longitudinal compressive force in the train; $\alpha_{u}$ - transverse unbalanced acceleration in the curve; $\gamma$ - slope of the working surface of the wheel flange to the plane of the track; $\lambda$ - prolate cycloid parameter.

The table also shows the values of the intermediate calculation parameters: $x_{1}$ - distance from the first axle of the truck to the pole of rotation; $b$ - contact prediction; $A_{0}$ - reduced contact area; $k_{\mathrm{w}}$ - coefficient that takes into account the wobble of the wheel set; $u_{\mathrm{M}}^{0}$ - total relative sliding of the point $M$ on the wheel flange; $Y_{1}$ - guiding force; $N$ - normal pressure at the point of contact; $\mu$ - coefficient of friction.

As a basic variant of the calculation, the curve with $R=400 \mathrm{~m} ; \gamma_{0}=60^{\circ} ; P_{w}=100 \mathrm{kN}$; $F=400 \mathrm{kN} ; \alpha_{\mathrm{u}}=0 ; \lambda=1,027 ; x_{1}=2,5 \mathrm{~m} ; \mu=0,25$ is considered.

Table 1. Calculation results of the wear factor $F_{\text {wear. }}$

\begin{tabular}{|c|c|c|c|c|c|c|c|c|c|c|c|c|c|c|c|}
\hline$R, \mathrm{~m}$ & $\gamma,{ }^{\circ}$ & $\begin{array}{c}P_{w}, \\
\mathrm{kN}\end{array}$ & $\begin{array}{c}F, \\
\mathrm{kN}\end{array}$ & $\begin{array}{c}\alpha_{\mathrm{u}}, \\
\mathrm{m} / \mathrm{s}^{2}\end{array}$ & $\lambda$ & $x_{1}, \mathrm{~m}$ & $A_{0}$ & $b, \mathrm{~m}$ & $\alpha, \mathrm{rad}$ & $k_{\mathrm{w}}$ & $U_{M}^{0}$ & $Y_{1}, \mathrm{kN}$ & $\mu$ & $N, \mathrm{kN}$ & $F_{\text {wear }}$ \\
\hline 400 & 60 & 100 & 400 & 0 & 1,027 & 2,5 & 0,813 & 0,0051 & 0,530 & & 0,535 & 90,5 & 0,25 & 122,1 & 20,1 \\
\hline 300 & 60 & 100 & 400 & 0 & 1,027 & 2,5 & 0,750 & 0,0069 & 0,560 & & 0,565 & 96,2 & 0,25 & 129,8 & 24,4 \\
\hline 500 & 60 & 100 & 400 & 0 & 1,027 & 2,5 & 0,850 & 0,0041 & 0,510 & & 0,515 & 86,5 & 0,25 & 116,8 & 17,7 \\
\hline 600 & 60 & 100 & 400 & 0 & 1,027 & 2,5 & 0,875 & 0,0034 & 0,496 & 0,8 & 0,401 & 83,5 & 0,25 & 112,7 & 12,9 \\
\hline 700 & 60 & 100 & 400 & 0 & 1,027 & 2,5 & 0,893 & 0,0029 & 0,485 & 0,6 & 0,294 & 81,1 & 0,25 & 109,5 & 9,0 \\
\hline 800 & 60 & 100 & 400 & 0 & 1,027 & 2,5 & 0,906 & 0,0026 & 0,476 & 0,4 & 0,193 & 79,2 & 0,25 & 106,9 & 5,7 \\
\hline 900 & 60 & 100 & 400 & 0 & 1,027 & 2,5 & 0,917 & 0,0023 & 0,469 & 0,2 & 0,095 & 77,5 & 0,25 & 104,6 & 2,7 \\
\hline 400 & 70 & 100 & 400 & 0 & 1,027 & 2,5 & 0,813 & 0,0082 & 0,580 & & 0,581 & 90,5 & 0,25 & 105,9 & 18,9 \\
\hline 400 & 60 & 100 & 400 & $-0,4$ & 1,027 & 2,7 & 0,798 & 0,0056 & 0,538 & & 0,543 & 78,5 & 0,25 & 105,9 & 18,0 \\
\hline 400 & 60 & 100 & 400 & $-0,2$ & 1,027 & 2,6 & 0,805 & 0,0053 & 0,534 & & 0,539 & 84,5 & 0,25 & 114,0 & 19,1 \\
\hline 400 & 60 & 100 & 400 & 0,2 & 1,027 & 2,4 & 0,820 & 0,0049 & 0,526 & & 0,531 & 96,5 & 0,25 & 130,2 & 21,1 \\
\hline 400 & 60 & 100 & 400 & 0,4 & 1,027 & 2,3 & 0,828 & 0,0047 & 0,522 & & 0,527 & 102,5 & 0,25 & 138,3 & 22,0 \\
\hline 400 & 60 & 100 & 400 & 0 & 1,01 & 2,5 & 0,813 & 0,0051 & 0,452 & & 0,452 & 90,5 & 0,25 & 122,1 & 17,0 \\
\hline 400 & 60 & 100 & 400 & 0 & 1,04 & 2,5 & 0,813 & 0,0051 & 0,586 & & 0,595 & 90,5 & 0,25 & 122,1 & 22,4 \\
\hline
\end{tabular}

\section{Analysis of the results}

From the analysis of the calculation data given in the table, it follows that when the radius of the curve varies from $R=300 \mathrm{~m}$ to $R=900 \mathrm{~m}$, all the design parameters included in formula (1) have a decreasing effect on the wear factor. In this case, $u_{\mathrm{M}}^{0}$ - total relative sliding decreases by 5.95 times, and $N$ - normal pressure at the point of contact decreases by 1.24 times. The wear factor $F_{\text {wear }}$ decreases by 9 times.

The variation in the value of the transverse unbalanced acceleration in the admissible limits for freight trains $\alpha_{\mathrm{u}}=0.3 \mathrm{~m} / \mathrm{s}^{2}$ [10] does not give the desired effect. When the transverse unbalanced acceleration changes from $\alpha_{\mathrm{u}}=0 \mathrm{~m} / \mathrm{s}^{2}$ to $\alpha_{\mathrm{u}}= \pm 0.3 \mathrm{~m} / \mathrm{s}^{2}$, the total relative sliding of the flange decreases by $1 \%$, the normal pressure in the contact of the flange with the rail increases by $10 \%$, as a result of which the wear factor also increases by $7 \%$.

Thus, decreasing the superelevation of the outer rail in the curve of small radius by 2 times, in accordance with formula (25), the wear rate of the side face of rails, in spite of the expectation of many specialists, does not decrease, but increases by $7 \%$. 


\section{Conclusion}

The paper presents a mechanical and mathematical model for estimating and predicting the wear rate of the side face of rails using the properties of a mathematical curve - cycloids depending on the operating characteristics of the track section and the parameters of the curves. This model has a great practical application.

Calculations by the presented model showed that in a curve with a radius of $400 \mathrm{~m}$ with an average velocity of $56 \mathrm{~km} / \mathrm{h}$, the superelevation of the outer rail of $h=100 \mathrm{~mm}$ should be made to obtain the transverse unbalanced acceleration $\alpha_{u}=0 \mathrm{~m} / \mathrm{s}^{2}$. When it decreases to $h=50 \mathrm{~mm}$, the value of unbalanced acceleration increases to $\alpha_{\mathrm{u}}=0.3 \mathrm{~m} / \mathrm{s}^{2}$. The wear rate increases by $7 \%$.

At the same time, there is an opinion that the intensive wear of rails in curves of small radius occurs because of the large values of the superelevation of the outer rail.

\section{References}

1. S.M. Andrievskii, Side wear of rails on curves (Transzheldorizdat, Moscow, 1959)

2. M.F. Verigo, Interaction of the track and rolling stock (Transport, Moscow, 1986)

3. A.Ya. Kogan, Dynamics of the track and its interaction with the rolling stock (Transport, Moscow, 1997)

4. I.N. Bronstein, Handbook of mathematics (Nauka, Moscow, 1980)

5. O.P. Ershkov, Calculations of transverse forces in curves (Transport, Moscow, 1966)

6. O.P. Ershkov, Studying of accumulation of railway track deterioration: Collection of scientific papers (Transport, Moscow, 1980)

7. S.V. Vershinskii, Dynamics, strength and stability of cars in heavy and high-speed trains: Collection of scientific papers (Moscow, 1970)

8. O.P. Ershkov, Increase of strength and reliability of the track: Collection of scientific papers (Transport, Moscow, 1989)

9. N.I. Karpushchenko, Rail transport operation 1, 14-17 (1996)

10. Instruction on the current maintenance of the railway track. Approved by the decree of JSC “Russian Railways” of 14.11.2016 № 2288 (JSC Russian Railways, Moscow, 2016) 